\title{
MODELAMIENTO DE UN ALGORITMO PARA LA ASIGNACIÓN DE LONGITUDES DE ONDA EN UN MULTIPLEXOR DWDM MINIMIZANDO EL PROCESO DE MEZCLADO DE LA CUARTA ONDA FWM (FOUR WAVE MIXING)
}

\section{MODELING OF AN ALGORITHM FOR ASSIGNING WAVELENGTHS IN DWDM MULTIPLEXER MINIMIZ- ING THE MIXING OF THE FOURTH WAVE FWM (FOUR WAVE MIXING)}

\begin{abstract}
One of the means of high-speed transmission most used in our environment is the optical fiber specified in ITU-T G.625 standard, but its deployment and use currently poses several challenges to solve due to its nonlinerarity characteristics, within which is the one related to the decreased level of interference. With the aim of providing a contribution to the solution of this problem is proposed to model the nonlinear behavior of FWM when implementing systems wavelength multiplexing of a single fiber optic strand in order to estimate the fundamental characteristics governing this technology and to propose an algorithm which is capable of reducing interference nonlinear fourth wave (FWM). As main conclusion it is found that the proposed model reduces interference nonlinear FWM, allowing an increase in the DWDM channel allocation increasing bandwidth provided in each optical link.
\end{abstract}

Key words: FWD, optical fiber, kerr efecto, WDM, fourth wave.

\section{RESUMEN}

Uno de los medios de transmisión de alta velocidad más utilizados en nuestro entorno es la fibra óptica especificada en el estándar ITU-T G.625; sin embargo, su despliegue y utilización plantea en la actualidad varios desafíos a resolver debido a su característica de no linealidad, dentro de los cuales se encuentra el relacionado con la disminución del nivel de interferencia. Con el ánimo de brindar un aporte en la solución de esta problemática se propone modelar el comportamiento no lineal de FWM cuando se implementan sistemas de multiplexación de longitudes de onda por un único hilo de fibra óptica a fin de poder estimar las características fundamentales que rige esta tecnología y poder plantear un algoritmo que sea capaz de reducir la interferencia no lineal de cuarta onda (FWM). Como conclusión principal se encontró que el modelo propuesto reduce la interferencia no lineal FWM, lo que permite un aumento en la asignación de canales DWDM aumentando el ancho de banda establecido en cada enlace óptico.

Palabras claves: FWD, fibra óptica, efecto kerr, WDM, cuarta onda.
Jorge Arturo Mojica Cáceres Magister en Ciencias de la Información y las Comunicaciones Universidad Católica de Colombia jamojicac@correo.udistrital.edu.co Bogotá, Colombia

Tipo: Artículo de investigación

Fecha de Recepción: Febrero 17 de 2013 Fecha de Aceptación: Mayo 2 de 2013 


\section{INTRODUCCIÓN}

En la actualidad existen miles de kilómetros de redes de fibra extendida por todo el mundo, los cuales permiten comunicar continentes y países enteros, sin embargo las demandas actuales por tener mayores anchos de banda que permitan el acceso a más servicios de datos a los usuarios finales, exigen que el medio de transmisión en sus troncales principales compuestas por fibra óptica mejore o amplíe la capacidad de transmisión, y es en este punto donde las técnicas multiplexación de longitudes de onda se establecen como una alternativa eficaz para incrementar la capacidad de transmisión y explotar a su máximo las redes de fibra actualmente instaladas. Sin embargo la multiplexación de longitudes de onda y la cantidad de canales asociados en un mismo hilo de fibra está sujeta a las características físicas de la fibra y su comportamiento lineal y no lineal al instante de transmitir un pulso de luz por este medio, razón por la cual, se plantea en la presente investigación unificar los conceptos matemáticos referentes al comportamiento del proceso no lineal FWM presente en las fibras ópticas cuando se implementan sistemas de multiplexación de longitudes de onda por un único hilo de fibra y se establece como enfoque fundamental estudiar el comportamiento de FWM en las fibras ópticas instaladas actualmente en nuestro medio, donde predominan las fibras óptica bajo estándar ITU-T G.652.D, con el fin de estimar los parámetros físicos y características fundamentales a tener en cuenta para el análisis y planteamiento de algoritmos de asignación de longitudes de ondas o canales DWDM minimizando efectos no lineales como el FWM en redes de fibra óptica ya instalada en nuestro país.

\section{PLANTEAMIENTODE LAINVESTIGACIÓN Y SU JUSTIFICACIÓN}

La fibra óptica es el medio de transmisión con mayores condiciones tecnológicas para la transferencia de datos de alta velocidad $(2,5 \mathrm{Gbps}$, $10 \mathrm{Gbps}$ o $40 \mathrm{Gbps}$ ) y es un factor primordial a tener en cuenta en la formulación de las nuevas tecnologías y arquitecturas de redes de comu- nicación.

Los sistemas de comunicación basados en fibra óptica han desarrollado técnicas de múltiplexación de longitudes de onda usadas para la transmisión de datos por un único hilo de fibra, esta tecnología es conocida como técnicas de multiplexacion WDM (Wavelength Division Multiplexing). Los sistemas WDM pueden combinar hasta 160 señales ópticas sobre una misma fibra, de este modo, cada una de las señales se pueden amplificar y transmitir simultáneamente asignándole a cada señal diferente longitud de onda, (que operan entre 1310nm y $1550 \mathrm{~nm}$ ), con el objeto de transmitir grandes cantidades de datos por un único medio a cientos de kilómetros de una forma económica y segura.

Cada señal representa un canal de comunicación y transmisión de datos, donde cada canal puede ser usado para trasmitir diferentes tipos de datos (video, voz, multimedia, entre otros).

Las técnicas de multiplexación por división de longitud de onda WDM, se dividen en dos grandes técnicas: CWDM (Coarse Wavelength Division Multiplexing) de aplicación económica y asignada principalmente a áreas metropolitanas y sistemas que manejen separaciones entre canales de $20 \mathrm{~nm}$, la segunda técnica es conocida como DWDM (Dense Wavelength Division Multiplexing) esta técnica multiplexa mayores cantidades de canales en una misma fibra óptica y permite la transmisión de grandes cantidades de datos estimadas en la actualidad en el orden de los TeraBit por segundo (Tbps) a cientos de kilómetros comunicando continentes y ciudades, esta técnica utiliza separaciones entre canales inferiores a $1 \mathrm{~nm}$.

Estas técnicas siguen evolucionando gracias a investigaciones impulsadas por diferentes organizaciones mundiales que dedican sus esfuerzos al mejoramiento de las condiciones de transmisión de datos por fibra óptica, con el propósito de incrementar los anchos de banda y las velocidades de transmisión. Los estudios e investigaciones realizadas al respecto en la actualidad se han enfocado en dos áreas principa-

Modelamiento de un algoritmo para la asignación de longitudes de onda en un multiplexor DWDM minimizando el proceso de mezclado de la cuarta onda FWM (four wave mixing) 
les: la primera, está dedicada al mejoramiento de las propiedades físicas de la fibra óptica al momento de su fabricación y la segunda área de investigación está enfocada a formular modelos matemáticos que contribuyan con la implementación y optimización de circuitos y/o dispositivos optoelectrónicos de multiplexación y transmisión de señales ópticas.

Dadas las condiciones tecnológicas de nuestro país en el campo de las telecomunicaciones por fibra óptica y los lineamientos de la industria del las telecomunicaciones en nuestro país, se plantea el desarrollo de una investigación que maneje los modelos matemáticos referentes a los comportamientos de las no linealidades de la fibra y su influencia en el espectro de las señales ópticas, considerando principalmente la influencia de las interferencias no lineales FWM presentes en la fibra y su influencia en el espectro óptico en pro del mejoramiento en el ancho de banda de las fibras monomodo de bajo costo (UIT-T G652) [1].

La presente investigación está orientada a buscar un modelamiento matemático que permita Generar un Algoritmo de Asignación de Longitudes de Onda que pueda ser implementado en un Multiplexor DWDM Minimizando la Modulación de la Four Wave Mixing, teniendo en cuenta el espaciamiento entre canales asignado al sistema DWDM, la potencia de las señales originales transmitidas, la cantidad de productos FWM generados en el sistemas de transmisión y la relación de estas tres variables con los parámetros de calidad de Transmisión de la señales ópticas en sistemas DWDM.

Téngase presente que FWM es un fenómeno no lineal que produce interferencias significativas entre canales, que se deben evitar que se deben evitar, pues esta interferencia no lineal impide utilizar al máximo el número de canales de un multiplexor DWDM.

Las interferencias FWM se generan cuando se usan tres longitudes de onda separadas simétricamente contiguas, con características de potencia y fase similares para la transmisión de datos, originando armónicas con la suficiente potencia para formar una cuarta onda que puede interferir con las características de amplitud, frecuencia y fase de otros canales originales establecidos en la transmisión del sistema WDM

Los alcances del trabajo contemplan recopilar y sintetizar los conceptos matemáticos que definen el fenómeno FWM con el fin de conceptualizar un modelo matemático que permita generar un procedimiento de asignación de longitudes de onda en un multiplexor DWDM con el fin de incrementar la utilización del ancho de banda de las fibras ópticas existentes minimizando el mezclado de la cuarta onda

Tras la unificación y síntesis de conceptos relacionados con el fenómeno FWM se busca generar un algoritmo de asignación de longitudes de onda en los sistemas de transmisión de datos basados en multiplexación WDM, a partir del análisis de los modelos y conceptos matemáticos estudiados para describir el mezclado de la cuarta onda, con el fin de reducir las interferencias no lineales presentes cuando se asignan canales con separación simétrica y usar el algoritmo para generar asignaciones de canales con espaciamiento asimétrico que permita minimizar la interferencia FWM.

\section{MODELAMIENTO DEL EFECTO KERR Y DEL MEZCLADO DE CUATRO ONDAS}

El efecto Kerr fue definido por John Kerr (1824 - 1907), el efecto electro-óptico, llamado efecto Kerr, genera fenómenos como el PMD (dispersión por modo de polarización) y la dependencia no lineal del índice de refracción respecto de la intensidad de la onda incidente genera los efectos no lineales SPM, XPM y FWM. El índice de refracción de la fibra óptica depende de la potencia de la señal transportada y esta dependencia se expresa mediante la ecuación (1).

$$
n=n_{0}+n_{2} \frac{P}{A_{g}}
$$

Donde $\mathrm{n}_{0}$ es el índice de refracción lineal o índice de refracción efectivo de la fibra óptica en ausencia de efecto no lineal, $\mathrm{n}_{2}$ es el índice de refracción no lineal un valor constante para 
fibra de silicio de $\mathrm{n}_{2} \cong 2,6 \times 10^{-20} \mathrm{~m}^{2} / \mathrm{W}$ y el cociente $\mathrm{P} / \mathrm{A}_{\mathrm{e}}$ corresponde a la intensidad de la onda incidente, potencia $\mathrm{P}$ sobre área efectiva $A_{e}$. Este efecto produce un ensanchamiento espectral del ancho del pulso. La contribución no lineal del índice de refracción genera un cambio de fase en la señal propagada de la forma $\phi_{\mathrm{NL}}=\gamma \mathrm{PL}_{\mathrm{e}}$, donde se define el coeficiente no lineal $\gamma$ mediante la ecuación (2).

$$
\gamma=\frac{2 \pi * f_{p} n_{2}}{c^{*} A_{e}}
$$

En donde c es la velocidad de la luz y $f_{p}$ es la frecuencia del pulso. Además la constante de propagación también se vuelve no lineal, dependiendo de la potencia aplicada (ecuación (3)).

$$
\beta_{N L}=\beta+\gamma P
$$

La ecuación (3) demuestra el efecto Kerr, donde se indica que a una alta intensidad de la onda se provocará un cambio en su constante de fase.

La ecuación no lineal de Shrodinger describe matemáticamente la propagación de los pulsos de luz en una fibra óptica (ecuación (4)).

$$
i u_{z}+\frac{D(z)}{2} u_{t t}+g(z)|u|^{2} u=0
$$

La ecuación (4) tiene soluciones estacionarias que permiten determinar equilibrios entre el efecto Kerr y la dispersión de la velocidad de grupo. Estos puntos de la ecuación se denominan solitones.

\subsection{Definición matemática del fenómeno no lineal FWM [2]}

El FWM o mezclado de cuatro ondas, es un fenómeno generado cuando se propagan varias ondas a frecuencias $\omega_{1}, \omega_{2}, \omega_{3}, \ldots, \omega_{n}$, por un mismo hilo de fibra óptica. La mezcla de cua- tro ondas se produce de forma general cuando tres portadoras de frecuencias distintas $\omega_{\mathrm{i}}, \omega_{\mathrm{j}}$ y $\omega_{k}$, con potencias $P_{i^{\prime}} P_{j}$ y $P_{k^{\prime}}$ interaccionan en el interior de la fibra para generar una cuarta onda. Esta cuarta onda se origina por la dependencia entre el índice de refracción de la fibra y la intensidad de la señales relación que genera la aparición de nuevos pulsos de señal con nuevas frecuencias o longitudes de onda, que obedecen la ecuación (5).

$$
\omega_{i j k}=\omega_{i} \pm \omega_{j} \pm \omega_{k}
$$

La generación de nuevos pulsos por efecto FWM, se deben considerar en sistema WDM, puesto que pueden producir degradaciones en los canales la trasmisión.

La consideración de este efecto es importante cuanto más próximos estén los canales del sistema WDM, por lo tanto en sistemas DWDM se debe analizar la relación espaciamiento entre canales y generación de pulsos por FWM para evitar diafonías.

Para comprender en detalle el efecto de FWM se considera un sistema de transporte de datos WDM con n canales donde el campo eléctrico se determina a partir de la ecuación (6).

$$
E(r, t)=\sum_{i=1}^{n} E_{i} \cos \left(\omega_{i} t-\beta_{i} z\right)
$$

Y la polarización no lineal PNL está dada por la ecuación (7).

$$
P_{N L}=\varepsilon_{0} X^{(3)} E^{3}
$$

Para el caso, la polarización no lineal se determina a partir de la ecuación (8).

El desarrollo de la ecuación (8) lleva al modelo descrio en la ecuación (9).

$$
P_{N L}=\varepsilon_{0} X^{(3)} \sum_{i=1}^{n} \sum_{j=1}^{n} \sum_{k=1}^{n} E_{i} \cos \left(\omega_{i} t-\beta_{i} z\right) E_{j} \cos \left(\omega_{j} t-\beta_{j} z\right) E_{k} \cos \left(\omega_{k} t-\beta_{k} z\right)
$$




$$
P_{N L}=\frac{3}{4} \varepsilon_{0} X^{(3)} \sum_{i=1}^{n} E_{i}^{2}+2 \sum_{j \neq i} E_{i} E_{j} E_{k} \cos \left(\omega_{i} t-\beta_{i} z\right)+\frac{1}{4} \varepsilon_{0} X^{(3)} \sum_{i=1}^{n} E_{i}^{3} \cos \left(3 \omega_{i} t-3 \beta_{i} z\right)+
$$

$$
\begin{gathered}
\frac{3}{4} \varepsilon_{0} X^{(3)} \sum_{i=1}^{n} \sum_{j \neq i} E_{i}^{2} E_{j} \cos \left\{\left(2 \omega_{i}-\omega_{j}\right) t-\left(2 \beta_{i}-\beta_{j}\right) z\right\}+\frac{3}{4} \varepsilon_{0} X^{(3)} \sum_{i=1}^{n} \sum_{j \neq i} E_{i}^{2} E_{j} \cos \left\{\left(2 \omega_{i}+\omega_{j}\right) t-\left(2 \beta_{i}+\beta_{j}\right) z\right\}+ \\
\frac{6}{4} \varepsilon_{0} X^{(3)} \sum_{i=1}^{n} \sum_{j>i} \sum_{k>j} E_{i} E_{j} E_{k} \cos \left\{\left(2 \omega_{i}+\omega_{j}+\omega_{k}\right) t-\left(\beta_{i}+\beta_{j}+\beta_{k}\right) z\right\}+\cos \left\{\left(\omega_{i}+\omega_{j}+\omega_{k}\right) t-\left(\beta_{i}+\beta_{j}+\beta_{k}\right) z\right\}+ \\
\cos \left\{\left(\omega_{i}-\omega_{j}+\omega_{k}\right) t-\left(\beta_{i}-\beta_{j}+\beta_{k}\right) z\right\}+\cos \left\{\left(\omega_{i}-\omega_{j}-\omega_{k}\right) t-\left(\beta_{i}-\beta_{j}-\beta_{k}\right) z\right\}
\end{gathered}
$$

En el desarrollo de la ecuación (9), aparece un conjunto de términos en los que las $n$ frecuencias presentes aparecen mezcladas de todas las formas posibles, tanto entre sí como consigo mismas. Esto significa que, los nuevos campos que se generarían tendrían frecuencias de la forma $\omega_{i} \pm \omega_{j} \pm \omega_{k}$, donde las correspondientes $\omega_{i}, \omega_{j}, \omega_{k}$ no han de ser necesariamente diferentes. La aparición de estas nuevas frecuencias, que surgen de la suma o resta de los valores de las tres iniciales, justifica el nombre que se le da a este fenómeno, Mezclado de cuatro ondas (FWM, por su nombre en inglés, Four Wave Mixing), ya que de tres de ellas surge una cuarta. Si esta cuarta frecuencia coincide en valor con alguna de las tres anteriores, aparecerá diafonía y será la causa de que sea preciso estudiar su posición para evitarlo.

Dado que los términos de la forma $\omega_{i}+\omega_{j}-\omega_{k}$ con $\mathrm{i}, \mathrm{j} \neq \mathrm{k}$, son los que, dan lugar a este fenómeno, serán a éstos términos a los que se haga una especial mención y se define mediante la ecuación (10).

$$
\omega_{i j k}=\omega_{i}+\omega_{j}-\omega_{k}
$$

Y se especifica un factor de degeneración d_ijk cuando se cumplan los parámetros establecidos por la ecuación (11).

$$
d_{i j k}+ \begin{cases}3, & i=j \\ 6, & i \neq j\end{cases}
$$

El término de la polarización dieléctrica no lineal, correspondiente a la frecuencia $\omega_{-}$ijk puede escribirse a partir de la ecuación (12).

$$
P_{N L}(z, t)=\frac{\varepsilon_{0} X^{(3)}}{4} d_{i j k} E_{i} E_{j} E_{k} \cos \left(\omega_{i j k} t-\beta_{i j k} z\right)
$$

A partir de la ecuación (12), y suponiendo que el área efectiva por la que propagan estos campos es $A_{e}$, puede demostrarse que la potencia generada a la frecuencia $\omega_{i j k}$, tras atravesar una distancia L viene dada por la ecuación (13).

$$
P_{i j k}=\left(\frac{\omega_{i j k} d_{i j k} X^{(3)}}{8 A_{e} n_{e f} c}\right)^{2} P_{i} P_{j} P_{k} L^{2}
$$

Donde $\mathrm{P}_{\mathrm{ijk}}$, son las potencias de entrada correspondientes a las frecuencias $\omega_{i j k}$, y se ha tomado el índice de refracción efectivo $n_{e f}$, del modo fundamental. En función del índice de refracción no lineal $n_{N L}$, podrá también escribirse como se plantea en la ecuación (14).

$$
P_{i j k}=\left(\frac{\omega_{i j k} n_{N L} d_{i j k}}{3 c A_{e}}\right)^{2} P_{i} P_{j} P_{k} L^{2}
$$

Con la ecuación (14) y para dar una idea del orden de magnitud de este fenómeno, supongamos que los tres canales presentes, cada uno con una frecuencia diferente, tiene una potencia de $1 \mathrm{~mW}$ y que el área efectiva es de $50 \mu \mathrm{m}^{2}$. Como los tres canales tienen diferentes frecuencias, $\mathrm{d}_{\mathrm{ijk}}=6$. Si se toma $\mathrm{n}_{\mathrm{NL}}=3 \times 10^{-8}$ $\mu \mathrm{m}^{2} / \mathrm{W}$, para una distancia $\mathrm{L}=20 \mathrm{~km}$, la potencia generada a la nueva cuarta frecuencia es de unos $9,5 \mu \mathrm{W}$, lo que implica que está a unos $20 \mathrm{~dB}$ por debajo de las potencias de las señales originales. Aunque este valor no es elevado, si en lugar de circular estos tres canales, también circula un cuarto canal con una frecuencia $\omega_{i j k}$, la diafonía puede degradar significativamente la calidad de transmisión del cuarto canal. 
En los análisis y cálculos anteriores se ha restringido al caso de que las tres frecuencias son diferentes, sin embargo este fenómeno aparece también en el caso que únicamente haya dos frecuencias distintas y las nuevas frecuencias o tono FWM que aparecerán están definidas mediante la ecuación (15).

$$
2 \omega_{1}-\omega_{2} \text { y } 2 \omega_{2}-\omega_{1}
$$

En general, si están presentes $\mathrm{N}$ canales, con $\mathrm{N}$ diferentes frecuencias, el número de nuevas señales $M$ que podrán aparecer vendrá dado por la ecuación (16).

$$
M=\frac{1}{2}\left(N^{3}-N^{2}\right)
$$

Esto hace que el efecto FWM sea tanto más pronunciado cuantos más canales estén presentes.

\section{MODELAMIENTO DEL ALGORITMO}

En el modelamiento del algoritmo de asignación de longitudes onda en sistemas WDM es necesario evaluar el comportamiento y condiciones técnicas del enlace relacionadas con los parámetros de potencia de transmisión/recepción, atenuación, dispersión y comportamiento de efectos no linealidades del enlace.

\subsection{Diseño del enlace de fibra óptica}

El cálculo del enlace de fibra óptica, define el valor de la atenuación del enlace y hace referencia a la atenuación total del cable o enlace considerando reservas (ecuación (17)).

$$
\alpha_{t}=L \cdot \alpha_{L}+n_{e} \cdot \alpha_{e}+n_{c} \cdot \alpha_{c}+\alpha_{r}
$$

Donde:

$L$ : longitud total del cable o fibra en Km.

$\alpha_{L}$ : coeficiente de atenuación de la fibra en $\mathrm{dB} / \mathrm{Km}$

$n_{e}:$ número de empalmes

$\alpha_{e}$ : atenuación por empalme

$n_{c}:$ número de conectores

$\alpha_{c}$ : atenuación por conector

$\alpha_{r}:$ reserva de atenuación en $\mathrm{dB} / \mathrm{Km}$.
Dentro del diseño y cálculos de enlace se debe considerar una reserva de atenuación o margen de enlace, para empalmes futuros pronosticados en términos de cortes de fibra o reparaciones y el pronóstico de la degradación de la fibra en su vida útil esto incluye una mayor degradación por absorción de grupos $\mathrm{OH}$. La magnitud de la reserva depende de la importancia del enlace y particularidades de la instalación e incidencias de corte de fibra posibles a presentarse en el enlace, normalmente se adoptan valores entre $0,1 \mathrm{~dB} / \mathrm{Km}$ y $0,6 \mathrm{~dB} / \mathrm{Km}$, sin embargo este valor estará sujeto a las condiciones de tendido de fibra óptica y al pronóstico de posibles incidencias de cortes de fibra que se puedan presentar en el enlace.

Las pérdidas en los empalmes se encuentran por debajo de $0,1 \mathrm{~dB} / \mathrm{Km}$ y en los conectores no superan $0,5 \mathrm{~dB} / \mathrm{Km}$, en los sistemas modernos de empalmes por fusión las pérdidas por empalme no superan los $0,02 \mathrm{~dB} / \mathrm{Km}$, de igual forma se debe considerar el umbral de sensibilidad del receptor para una tasa de error de bit (BER) especifica que es la mínima cantidad de potencia óptica necesaria para que el equipo óptico receptor obtenga el BER deseado dentro del sistema digital. En los sistemas analógicos es la mínima cantidad de potencia de luz necesaria para que el equipo óptico obtenga el nivel de señal a ruido $(\mathrm{S} / \mathrm{N})$ deseado.

\subsection{Cálculos de interferencia no lineal FWM [3]}

Dado el conjunto $\mathrm{Z}$ de $\mathrm{N}$ Canales definido como $Z=\left\{\omega_{1}, \omega_{2}, \omega_{3}, \ldots, \omega_{N}\right\}$ a implementar en un sistema WDM, se calcula la cantidad M de posibles componentes de frecuencia interferentes generados por FWM mediante la ecuación (16).

Cada canal o pulso generado por efecto FWM se establece con una nueva frecuencia o longitud de onda, que obedecen a la ecuación (10).

Donde $k \neq i, j$ y $k, i, j$ toman valores de $1,2,3, \ldots, \mathrm{N}$.

La potencia óptica $P_{i j k}$ del canal o pulso generado por FWM $\left(\omega_{i j k}\right)$, se establece al final del enlace mediante la ecuación (18). 


$$
P_{i j k}=\left(\frac{D_{i j k}}{3} \gamma L_{e}\right)^{2} P_{i} P_{j} P_{k} e^{-\alpha L} \eta
$$

Donde:

$D_{i j k}=3$ si $i=j$ determinado como caso degenerado.

$D_{i j k}=6$ si $i \neq j \neq k$ determinado como caso no degenerado.

$\gamma$ : coeficiente no lineal $\left(\gamma=2 \pi n_{2} / \lambda A_{e}\right)$.

$n_{2}$ : es el índice de refracción no lineal que para fibras de sílice es $2,6 \times 10^{-20} \mathrm{~m}^{2} / \mathrm{W}$.

$A_{e}$ : área eficiente del modo de propagado bajo consideración.

$L_{e}$ : longitud efectiva de interacción no lineal de la fibra óptica $\left(L e=\left(1-e^{-\alpha L}\right) / \alpha\right)$.

$\alpha$ : coeficiente de pérdidas no nulo de la fibra óptica.

$L$ : longitud del enlace óptico.

$P_{i}, P_{j}, P_{k}$ : potencias de los canales considerados en FWM.

$\eta$ : eficiencia de generación de FWM. Se calcula mediante la ecuación (19).

$\eta=\left(\frac{\alpha^{2}}{\alpha^{2}+\Delta \beta^{2}}\right)\left(1+\frac{4 e^{-\alpha L} \sin ^{2}(\Delta \beta L / 2)}{\left(1-e^{-\alpha L}\right)^{2}}\right)$

Donde, el parámetro $\Delta \beta=\beta_{1}+\beta_{2}-\beta_{3}-\beta_{4}$ es la relación de desfases ópticos en la propagación de las cuatro ondas consideradas, para valores de $\Delta \beta$ cercanos a 0 se define como condición de fase, y determinan que la eficiencia tiende a su valor máximo $\eta=1$ y por lo tanto de los tonos generados por FWM, para encontrar el valor de $\Delta \beta$ se utiliza la ecuación (20).

$$
\Delta \beta=\frac{2 \pi \lambda_{0}^{2}}{C}\left(f_{i}-f_{k}\right)\left(f_{j}-f_{k}\right)\left[D\left(\lambda_{0}\right)-\frac{\lambda_{0}^{2}}{C}\left(\frac{f_{i}+f_{j}}{2}-f_{i}\right) S\left(\lambda_{0}\right)\right]
$$

Donde, $\mathrm{D}\left(\lambda_{0}\right)$ (ps/nmkm) es el valor de la dispersión y $S\left(\lambda_{0}\right)=d D / d \lambda\left(\mathrm{ps} / \mathrm{nm}^{2} \mathrm{~km}\right)$ es la pendiente de dispersión. $\lambda_{0}$, es la longitud de onda donde se consideran los valores de la dispersión y su pendiente.

Dado que sobre un canal original $\omega_{s}$ establecido en el conjunto $\mathrm{Z}$ del sistema WDM a implementar puede recaer múltiples tonos o pulsos generados por efecto FWM producto de las diferentes combinaciones de tonos, canales o frecuencias originales del sistema WDM, se deben calcular todas las interferencias FWM presentes en el canal $\omega_{s}$ por las múltiples combinaciones de canales. La participación de las diferentes interferencias FWM producto de cada combinación de canales sobre el canal $\omega_{s}$ se puede establecer de la siguiente forma:

- La sumatoria de todas las interferencias FWM producto de combinaciones cuando todos los canales son diferentes $i \neq j \neq k \neq s$.

- La sumatoria de todas las interferencias FWM producto de combinaciones cuando todos los canales $i, j, k$ son diferentes pero $\mathrm{k}$ toma el valor del canal interferido $s$ esto es $i \neq j \neq k \neq s$.

- La sumatoria de todas las interferencias FWM producto de las combinaciones cuando los canales $i, j$ son iguales y $\mathrm{k}$ es diferente de $i, j$. Adicionalmente $i, j, k$ son diferente de $s$; esto es $i=j \neq k$ con $i, j, k \neq s$.

Con lo anterior se establece que la potencia de ruido generado por FWM en el detector WDM se obtiene a partir de la ecuación (21) [4].

$\sigma_{F W M}^{(1) 2}=2 K^{2} P_{s}\left\{\frac{1}{8} \sum_{i \neq j \neq k \neq s} P_{i j k}+\frac{1}{4} \sum_{i \neq j \neq k=s} P_{i j k}+\frac{1}{4} \sum_{i=j \neq k} P_{i j k}\right\}$

El factor de calidad $q$ del sistema se establece con la implementación de la ecuación (22).

$$
q=\frac{K P_{s}}{\sqrt{\sigma_{F W M}^{(1) 2}+\sigma_{t h}^{2}+\sigma_{s h}^{2}+\sqrt{\sigma_{t h}^{2}}}}
$$

De la anterior expresión se puede despejar la potencia óptica $P_{s}$ que permite mantener el nivel de calidad $q$ en el receptor, si se relaciona el valor $q$ con la probabilidad de error BER, así como la potencia $P_{S o}$, necesaria en el receptor para mantener el mismo nivel de calidad q sin considerar la degradación de FWM, al comparar estas potencias se obtiene la penalización de potencia por FWM establecida por la ecuación (23). 


$$
\begin{gathered}
\delta_{F W M}=10 \log \left(\frac{P_{s}}{P_{s 0}}\right) \\
\delta_{F W M}=10 \log \left[\frac{1}{1-2 q^{2} C_{F W M}^{(1)}}\right]
\end{gathered}
$$

El valor de $\mathrm{C}_{\mathrm{FWM}}$ se obtiene de la ecuación (24).

$C_{F W M}^{(1)}=\left\{\frac{1}{8} \sum_{i \neq j \neq k \neq s} \frac{P_{i j k}}{P_{s}}+\frac{1}{4} \sum_{i \neq j \neq k=s} \frac{P_{i j k}}{P_{s}}+\frac{1}{4} \sum_{i=j \neq k} \frac{P_{i j k}}{P_{s}}\right\}$

Para evaluar la penalización de un sistema WDM de múltiples canales se debe hacer el cálculo de todos los productos generados por FWM que producen interferencia en el canal s, la potencia de estos productos se calcula por la ecuación (25).

$$
P_{i j k}=\left(\frac{D_{i j k}}{3} \gamma L_{e}\right)^{2} P_{i} P_{j} P_{k} e^{-\alpha L} \eta
$$

En los diseños de sistemas de telecomunicaciones el rango para el BER se establece en valores de $10^{-9}$ a $10^{-12}$, para los cálculos de transmisión por fibra óptica se toma el valor de $10^{-12}$. El BER está muy relacionado con el factor Q (figura 1), esta relación se presenta mediante la ecuación (26).

$$
\begin{gathered}
B E R=P_{e}(Q) \\
B E R=\frac{1}{\sqrt{\pi}} \int_{q / \sqrt{2}}^{\infty} e^{-x^{2}} d x \\
B E R=\frac{1}{2}\left[1-\operatorname{erf} \frac{Q}{\sqrt{2}}\right] \\
B E R \approx \frac{1}{\sqrt{2 \pi}} \frac{e^{-Q^{2} / 2}}{Q}
\end{gathered}
$$

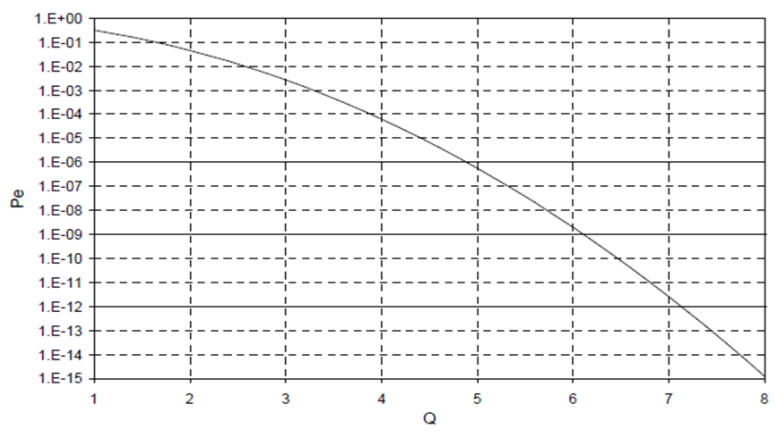

Figura 1. Probabilidad error (Pe, BER) Vs Factor Q [5].
De la grafica anterior, para obtener un $P_{e}=10^{-9}$ se requerirá un factor $\mathrm{Q}=6$ y para obtener un $P_{e}=10^{-12}$ se requiere un factor $\mathrm{Q}=7$.

\subsection{Planteamiento del algoritmo}

Se selecciona el cuadro de canales o rango de frecuencias y/o longitudes de onda a asignar con igual separación entre ellos. Estos canales deben estar normalizados y acorde a lo dispuesto en la norma ITU-T G.694.1 (DWDM) se ordenan los canales de mayor a menor frecuencia (f) o de menor a mayor longitud de onda $(\lambda)$.

Se elige los dos primeros canales que dan inicio al cálculo para seleccionar canales con baja interferencia FWM. En principio estos canales pueden ser cualquiera ubicados en el rango especificado en el numeral anterior, se asigna $\mathrm{C}_{1}$ como canal $1 \mathrm{y} \mathrm{C}_{2}$ como canal 2; para efectos del algoritmo estas variables se refieren a la $\mathrm{f}_{1} \mathrm{y} \mathrm{f}_{2}$, cuyos valores de estas dos frecuencias preferiblemente se escogen los dos canales de mayor frecuencia (f) o menor longitud de onda $(\lambda)$.

Se selecciona un tercer canal denominado $\mathrm{C}_{3}$, el cual puede tomar los valores de frecuencia o longitud de onda de los canales que están ubicados en cualquiera de los rangos especificado en el numeral uno, sin embargo y para tener un análisis detallado del comportamiento del tercer canal y tener una evaluación del comportamiento de las interferencias FWM al escoger un canal $\mathrm{C}_{3}$ e involucrarlo en la transmisión WDM, se asigna a $\mathrm{C}_{3}$ el valor de la siguiente frecuencia adyacente a $\mathrm{C}_{2}$ y que pertenece al rango especificado en el numeral uno.

Se calculan las posibles interferencia FWM producto de la asignación del nuevo canal $\mathrm{C}_{3}$ utilizando la siguiente metodología:

- Se realiza la evaluación FWM de los canales seleccionados $\mathrm{C}_{1}, \mathrm{C}_{2}$ y $\mathrm{C}_{3}$, de esta forma se evalúan todos los productos generados FWM con la selección de canales $\mathrm{C}_{1}, \mathrm{C}_{2} \mathrm{y} \mathrm{C}_{3}$. Luego se calcula el valor de $\mathrm{M}$ a partir de la ecuación (16), donde, $\mathrm{N}=3$ y M es el total de productos a analizar, y cada produc-

Modelamiento de un algoritmo para la asignación de longitudes de onda en un multiplexor DWDM minimizando el proceso de mezclado de la cuarta onda FWM (four wave mixing) 
to tendrá un valor $\omega_{\mathrm{ijk}}$ que se encuentran cumpliendo con el parámetro de combinación de frecuencias descrito en la ecuación (10); donde $k \neq i, j$ y $k, i, j$ toman valores en el rango $\{1,2,3\}$.

- Encontradas todas las combinaciones $\omega_{i j k}=\omega_{i}+\omega_{j}-\omega_{k}$ con las condiciones del numeral anterior se procede a calcular la interferencia FWM para definir si el valor de frecuencia seleccionado para fijar canal $\mathrm{C}_{3}$, permite la mínima interferencia FWM entre canales, esto significa verificar que la interferencia generada por los nuevos productos FWM en cada canal no superen la penalización de $1 \mathrm{~dB}$, de lo contrario se deberá desechar el valor asignado de frecuencia al canal $\mathrm{C}_{3}$ y tomar el siguiente valor de la tabla o rango especificado en el numeral uno.

- La metodología matemática para verificar que la penalidad FWM generada en cada canal $\mathrm{C}_{1}, \mathrm{C}_{2} \mathrm{y} \mathrm{C}_{3}$ asignado al sistema WDM no supere $1 \mathrm{~dB}$ se establece con el cálculo de la ecuación (23), la ecuación establece que para un BER $10^{-12}$ se debe tomar un $\mathrm{q}=7$ y si asignamos que la máxima penalidad $\delta_{\mathrm{FWM}}$ por efecto FWM es de $1 \mathrm{~dB}$ se deduce que $\mathrm{C}_{\mathrm{FWM}}{ }^{1}$ debe tomar valores menores a 0,0021. Tomando estos parámetros se establece que el punto de evaluación es $\mathrm{C}_{\mathrm{FWM}}{ }^{1} \leq 0,0021$ y se calcula bajo la ecuación (24).

- Se debe tener presente que hasta este momento $k, i, j, s$ toman valores $\{1,2,3\}$ y s representa el valor de la frecuencia del canal original que para el caso son los valores de $\mathrm{C}_{1}$ ó $\mathrm{C}_{2}$ ó $\mathrm{C}_{3}$.

- Para evaluar la penalización se debe hacer el cálculo de $P_{S}$ el cual corresponde a la potencia del canal interferido al final del enlace o de entrada al receptor; adicionalmente se calculan todos los productos generados por FWM que producen interferencia en el canal s, la potencia de estos productos se calcula mediante la ecuación (25).

Para asignar el canal $3\left(\mathrm{C}_{3}\right)$, se debe tener presente que la distancia entre el canal $\mathrm{C}_{2} \mathrm{y} \mathrm{C}_{3}$ debe ser diferente a la distancia entre canal $\mathrm{C}_{1} \mathrm{y} \mathrm{C}_{2}$ esto con el fin de reducir la acumula- ción de interferencias FWM, por lo tanto para la asignación del $\mathrm{C}_{3}$ iniciamos dejando un canal de diferencia entre $\mathrm{C}_{2}$ y $\mathrm{C}_{3}$ (un canal intermedio). Para la asignación de posteriores canales se recomienda dejar de manera aleatoria una distancia entre canales a asignar (canales intermedios) que varíen entre cero canales y tres canales de distancia, esto se hace de manera aleatoria buscando no tener patrones de repetición entre asignaciones de canales con el espaciamiento entre los mismo.

Una vez se especifica el valor de frecuencia del canal 3, se procede a seleccionar la frecuencia para establecer el valor del siguiente canal $\mathrm{C}_{4}$, para lo cual se ajusta que $\mathrm{N}=4$ y el conjunto de valores que toman $k, i, j$, s son $\{1,2,3,4\}$, con estos nuevos parámetros y fijados los valores de

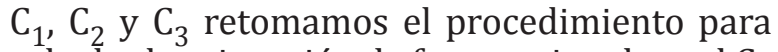
calcular la asignación de frecuencias al canal $\mathrm{C}_{4}$ a partir del numeral cuatro (4).

De manera general se establece que para evaluar el valor del siguiente canal $n\left(C_{n}\right)$, se selecciona una nueva frecuencia establecida en el rango restante no evaluado fijado en el numeral uno, ajustando en cada nueva asignación de $\mathrm{Cn}$ los valores $\mathrm{N}=\mathrm{N}+1$, y el conjunto de valores que toman k,i,j,s se extendería hasta $\{1,2,3,4, \ldots, \mathrm{n}\}$, con estos nuevos parámetros y fijados los valores de $C_{1}, C_{2}, C_{3}, \ldots, C_{n-1}, C_{n}$, retomamos el procedimiento para calcular la asignación de frecuencias al canal $\mathrm{C}_{\mathrm{n}}$ a partir del numeral cuatro, siempre que el rango de evaluación establecido en el numeral uno, tenga frecuencias para evaluar, de lo contrario se establecerá que al terminar el rango de frecuencias establecido en el numeral uno, se termina la posibilidad de asignar más canales y por lo tanto se determinará según el caso que el sistema WDM puede usar un máximo de canales $\mathrm{C}_{\mathrm{n}-1}$ ó $\mathrm{C}_{\mathrm{n}}$.

\section{PRESENTACION DE RESULTADOS}

\subsection{Presentación de condiciones iniciales}

Se evalúa el comportamiento de los productos FWM en una grilla ITU-T G.694.1 (frecuencia central $193.1 \mathrm{THz}$ ) con espaciamiento simétrico $193,1 \pm n \times 0,0125$, encontrado que la máxima 
frecuencia de la grilla se establece en 195,9375 $\mathrm{THz}$ equivalente a una longitud de onda de 1530,04 nm y la mínima frecuencia de la grilla es de $184,4875 \mathrm{THz}$ equivalente a una longitud de onda $1625 \mathrm{~nm}$, en total bajo este rango se establecen 917 canales de Operación.

Tomando como base este cuadro de canales se procede a asignar los canales de operación que presentan cero interferencias FWM entre ellos, este procedimiento se lleva a cabo evaluando todas las combinaciones en la ecuación (10).

Donde, $k \neq i, j y k, i, j$ toman valores de $\{1,2,3, \ldots, \mathrm{N}\}$ asegurando que cada nuevo canal a asignar no genere interferencia $\omega_{i j k}$ en los canales ya asignados, con anterioridad. Para nuestro caso evaluaremos la posibilidad de asignar 16 canales de transmisión con cero interferencia FWM entre ellos.

En el proceso para establecer el funcionamiento del algoritmo de asignación de longitudes de onda en un sistema WDM, se tomó como primer canal del sistema WDM la frecuencia de $195,9375 \mathrm{THz}$ y a partir de esta frecuencia se evaluó la asignación de cada uno de los 15 canales restantes para generar el patrón de evaluación del algoritmo de asignación de longitudes de onda generado en este trabajo de investigación.

Tras una evaluación minuciosa al asignar frecuencias a cada uno de los 16 canales y un análisis de la generación de productos FWM, se estableció que para asignar los 16 canales con cero interferencia FWM entre canales originales WDM, se requiere contar con una grilla de 252 frecuencias espaciadas simétricamente acorde a lo establecido y planteado por la UIT-T en su recomendación G.694.1 con espaciamiento $193,1 \pm n \times 0,0125$, obteniendo como patrón de evaluación y punto de referencia para el algoritmo generado en este trabajo de investigación la tabla I de asignación de canales.

Estableciendo la tabla 1 como patrón o guía para demostrar la importancia del planteamiento de nuestro algoritmo, también se generó un patrón gráfico de referencia que permi- tiera determinar gráficamente la importancia e impacto y los beneficios del planteamiento del algoritmo de asignación de longitudes de onda en sistemas DWDM.

Tabla 1. Longitudes de onda asignadas a 16 canales con cero interferencias FWM.

\begin{tabular}{|c|c|c|c|}
\hline $\begin{array}{c}\text { Canales a } \\
\text { asignarles } \\
\text { Frecuencia de } \\
\text { operación }\end{array}$ & $\begin{array}{c}\text { Canal } \\
\text { Utilizado } \\
\text { UIT-T } \\
\text { G.651.1 }\end{array}$ & $\begin{array}{c}\text { Frecuencia } \\
\mathrm{f}(\mathrm{THz})\end{array}$ & $\begin{array}{c}\text { Longitud } \\
\text { de Onda } \\
\lambda(\mathrm{nm})\end{array}$ \\
\hline $\begin{array}{c}\text { CANAL 1 } \\
\text { ASIGNAD0 }=\end{array}$ & $\mathrm{C} 1$ & 195,9375 & 1529,82 \\
\hline $\begin{array}{c}\text { CANAL 2 } \\
\text { ASIGNAD0 }=\end{array}$ & $\mathrm{C} 2$ & 195,9250 & 1529,92 \\
\hline $\begin{array}{c}\text { CANAL 3 } \\
\text { ASIGNAD0 }=\end{array}$ & $\mathrm{C} 4$ & 195,9000 & 1530,12 \\
\hline CANAL 4 & $\mathrm{C} 8$ & 195,8500 & 1530,52 \\
\hline
\end{tabular}

\begin{tabular}{c|c|c|c|}
$\begin{array}{c}\text { CANAL 4 } \\
\text { ASIGNAD0 }\end{array}$ & C8 & 195,8500 & 1530,52 \\
\hline $\begin{array}{c}\text { CANAL 5 } \\
\text { ASIGNAD0 }=\end{array}$ & C13 & 195,7875 & 1531,02 \\
\hline $\begin{array}{c}\text { CANAL 6 } \\
\text { ASIGNAD0 }=\end{array}$ & C21 & 195,6875 & 1531,82 \\
\hline $\begin{array}{c}\text { CANAL 7 } \\
\text { ASIGNAD0 }=\end{array}$ & C31 & 195,5625 & 1532,82 \\
\hline $\begin{array}{c}\text { CANAL 8 } \\
\text { ASIGNAD0 }=\end{array}$ & C45 & 195,3875 & 1534,22 \\
\hline $\begin{array}{c}\text { CANAL 9 } \\
\text { ASIGNAD0 }=\end{array}$ & C66 & 195,125 & 1536,32 \\
\hline $\begin{array}{c}\text { CANAL 10 } \\
\text { ASIGNAD0 }=\end{array}$ & C81 & 194,9375 & 1537,82 \\
\hline $\begin{array}{c}\text { CANAL 11 } \\
\text { ASIGNAD0 }=\end{array}$ & C97 & 194,7375 & 1539,42 \\
\hline $\begin{array}{c}\text { CANAL 12 } \\
\text { ASIGNAD0 }=\end{array}$ & C123 & 194,4125 & 1542,02 \\
\hline $\begin{array}{c}\text { CANAL 13 } \\
\text { ASIGNAD0 }=\end{array}$ & C148 & 194,1000 & 1544,52 \\
\hline $\begin{array}{c}\text { CANAL 14 } \\
\text { ASIGNAD0 }=\end{array}$ & C182 & 193,6750 & 1547,92 \\
\hline $\begin{array}{c}\text { CANAL 15 } \\
\text { ASIGNAD0 }=\end{array}$ & C204 & 193,4000 & 1550,12 \\
\hline $\begin{array}{c}\text { CANAL 16 } \\
\text { ASIGNAD0 }=\end{array}$ & C252 & 192,8000 & 1554,92 \\
\hline
\end{tabular}

En la figura 2 se establece que los canales que toman un valor de tres en el eje $\mathrm{Y}$, son los canales asignados al sistema WDM y los canales que toman un valor de 0,5 son los canales que no se pueden usar en el sistema de transmisión dado que pueden generar interferencia o crosstalk

Modelamiento de un algoritmo para la asignación de longitudes de onda en un multiplexor DWDM minimizando el proceso de mezclado de la cuarta onda FWM (four wave mixing) 
lineal.

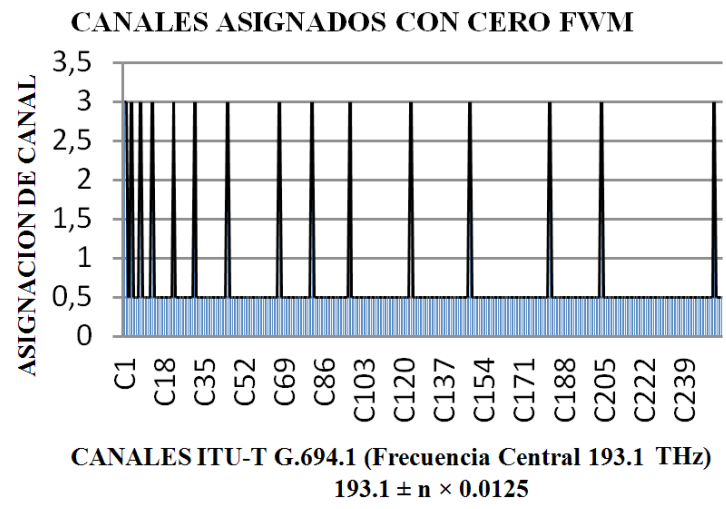

Figura 2. Canales asignados con cero Interferencias generados FWM.

Como se puede observar se requieren 252 canales de una grilla ITU-T G.694.1 (Frecuencia Central 193,1 THz) con espaciamiento simétrico $193,1 \pm n \times 0,0125$, para establecer 16 canales de operación con cero interferencia FWM, esto indica que el 93\% de los canales, frecuencias o longitudes de onda establecidas por G.694.1 bajo espaciamiento simétrico $193.1 \pm$ $\mathrm{n} \times 0.0125$ requeridos para establecer estos 16 canales no se podrán usar para transmisión de datos.

Ahora bien, establecida la grilla o cantidad de canales a evaluar se plantean las características y condiciones del o de los enlaces ópticos sobre los cuales se prueba el algoritmo planteado.

Dado el interés de determinar el comportamiento del algoritmo en las asignaciones de longitudes de onda o frecuencias en multiplexores DWDM, se establecen los parámetros y variables a utilizar e implementar en el algoritmo principalmente enfocado a usar enlaces de fibra óptica ITU-T G.652.D.

En la tabla 2 se especifican las condiciones y características del enlace óptico a establecer en el algoritmo.

\subsection{Análisis de resultados}

Con la aplicación del algoritmo planteado para aumentar el número de canales a asignar en la grilla de 252 frecuencias establecidas y bajo las condiciones y características de enlace formuladas anteriormente se procede a recalcular la asignación de los 16 canales inicialmente formulados.

Durante el proceso de cálculos de asignación de longitudes de onda y análisis de las potencias de cada tono FWM generado, conforme a lo planteado en el algoritmo propuesto se encuentra que la potencia de los tonos generados y la relación $\mathrm{P}_{\mathrm{ijk}} / \mathrm{P}_{\mathrm{s}}$ no permiten cumplir con las condiciones de penalización FWM y específicamente que no se pueda cumplir con la condición limite de $\mathrm{C}_{\mathrm{FWM}} \leq 0,00210$, para asignar canales que generen tonos interferentes FWM con baja potencia y que no superen el valor de penalización $\delta_{\mathrm{FWM}}=1 \mathrm{~dB}$.

Caso de asignación canal 5. Se asigna una posible frecuencia de la grilla 195,8375 THz correspondiente a $\mathrm{C} 9$ encontrando interferencias con los canales asignados $\mathrm{C}_{8}, \mathrm{C}_{2}, \mathrm{C}_{1}$ e incluso productos que auto interfieren el posible $\mathrm{C}_{9}$, en total se generan 8 interferencias de canales representados en dos interferencias al canal $\mathrm{C}_{8}$, dos interferencias al canal $\mathrm{C}_{9}$, dos interferencias al canal $\mathrm{C}_{1}$ y dos interferencias al canal $\mathrm{C}_{2}$, cada una de estas interferencias presenta una relación $\mathrm{P}_{\mathrm{ijk}} / \mathrm{P}_{\mathrm{s}}=0,03339204$. Dado que los valores de la relación $\mathrm{P}_{\mathrm{ijk}} / \mathrm{P}_{\mathrm{s}}$, no permiten obtener un esta relación $\mathrm{C}_{\mathrm{FWM}} \leq 0,00210$ el canal $\mathrm{C}_{9}$ propuesto como canal 5 se descarta y se procede con la evaluación del canal $\mathrm{C}_{10}$.

En general, este fenómeno o caso especifico explicado anteriormente, se presenta durante toda la evaluación y desarrollo del algoritmo para la asignación de los canales hasta llegar al canal 16, encontrando que el algoritmo planteado no mejora las condiciones de asignación de longitudes de onda especificadas inicialmente. Tras un análisis pormenorizado de las variables y características de los enlaces ópticos se encontró que la variable (L), que especifica la longitud del enlace es la que presenta una mayor incidencia en el comportamiento de la evaluación de la penalización FWM parámetro esencial para determinar el uso del canal asignado. Motivo por el cual se centro la aten- 
ción sobre la variable longitud de enlace (L), para evaluar la afectación de la penalización FWM encontrando que el algoritmo permite su aplicabilidad en enlaces que superan los 44 $\mathrm{Km}$, pues en enlaces inferiores a esta distancia con las características del enlace óptico establecido, las interferencias FWM superan las penalizaciones estipuladas para este fenómeno y no permiten mejorar la asignación de los 16 canales, tal como se explicó para el Caso de Asignación canal 5.

Tras el análisis encontrado se procede a parametrizar nuevamente la longitud del enlace óptico modificando la variable $L$ a un valor de $70 \mathrm{Km}$ y se mantienen todas las condiciones técnicas iniciales establecidas en la tabla 2.

Se procede nuevamente a recalcular y a aplicar el algoritmo para demostrar su funcionalidad encontrando los siguientes resultados.

El algoritmo se ejecuta para asignar nuevamente los 16 canales señalados inicialmente encontrando que la asignación de estos 16 canales se puede hacer con espaciamiento simétrico, esta asignación se establece con la distribución especificada en la tabla 3.

Como podemos observar, la asignación de canales es prácticamente continua en la grilla establecida por la UIT-T en su recomendación G.694.1 con espaciamiento $193,1 \pm n \times 0,0125$, el algoritmo planteado determina que pueden usarse los canales continuos entre el canal uno y el canal 16, sin embargo se debe anotar que entre cada iteración de asignación de canales continuos genera tonos nuevos de interferencia FWM, esta condición origina serias dificultades para hacer analizar tono por tono y determinar el impacto de cada uno de ellos en los canales originales ya existentes.

En la tabla 4 se especifica el crecimiento y cantidad de tonos interferentes FWM que se generan y se hacen presentes en la asignación de cada uno de los 16 canales.
Tabla 2. Características y condiciones del enlace óptico.

\begin{tabular}{|c|c|c|c|}
\hline Descripción & Nomenclatura & Valor & Unidades \\
\hline $\begin{array}{l}\text { Referencia } \\
\text { Tipo de } \\
\text { Fibra para } \\
\text { el Enlace } \\
\end{array}$ & ITU-T G.652.D & \multicolumn{2}{|c|}{ Ref.: Fibras Allwave } \\
\hline $\begin{array}{l}\text { Lambda de } \\
\text { Operación }\end{array}$ & $\lambda 0$ & 1550 & $\mathrm{~nm}$ \\
\hline $\begin{array}{l}\text { Área } \\
\text { Efectiva de } \\
\text { la Fibra }\end{array}$ & $\mathrm{Ae}$ & 80 & $\mu \mathrm{m}^{2}$ \\
\hline $\begin{array}{l}\text { Penalidad } \\
\text { FWM de } 1 \\
\text { dB }\end{array}$ & $\mathrm{C}_{\mathrm{FWM}}$ & $\leq 0,00210$ & \\
\hline $\begin{array}{l}\text { Penalidad } \\
\text { FWM de 0,5 } \\
\text { dB }\end{array}$ & $\mathrm{C}_{\mathrm{FWM}}$ & $\leq 0,00111$ & \\
\hline Índice de & & & \\
\hline
\end{tabular}

\begin{tabular}{|c|c|c|c|}
\hline \begin{tabular}{|l|} 
Indice de \\
Refracción \\
no lineal del \\
Sílice
\end{tabular} & $\mathrm{n} 2$ & $2,6 \mathrm{E}-20$ & $\mathrm{~m}^{2} / \mathrm{W}$ \\
\hline $\begin{array}{l}\text { Pendiente } \\
\text { de } \\
\text { dispersión } \\
\text { de la fibra }\end{array}$ & $S(\lambda 0)$ & 0,087 & $\mathrm{ps} / \mathrm{m}^{2} \mathrm{Km}$ \\
\hline $\begin{array}{l}\text { Dispersión } \\
\text { de la fibra a } \\
(\lambda 0)\end{array}$ & $\mathrm{D}(\lambda 0)$ & 17 & Ps/nmKm \\
\hline $\begin{array}{l}\text { Velocidad } \\
\text { de la Luz }\end{array}$ & c & 299792458 & $\mathrm{~m} / \mathrm{s}$ \\
\hline $\begin{array}{l}\text { Atenuación } \\
\text { de la fibra a } \\
(\lambda 0)\end{array}$ & $\alpha$ & 0,2 & $\mathrm{~dB} / \mathrm{Km}$ \\
\hline $\begin{array}{l}\text { Longitud } \\
\text { del enlace }\end{array}$ & $\mathrm{L}$ & 40 & $\mathrm{Km}$ \\
\hline $\begin{array}{l}\text { Perdidas } \\
\text { por } \\
\text { Empalme } \\
\end{array}$ & $\mathrm{Pe}$ & 0,02 & $\mathrm{~dB}$ \\
\hline $\begin{array}{l}\text { Perdidas } \\
\text { por } \\
\text { Conectores } \\
\end{array}$ & Pc & 0,4 & $\mathrm{~dB}$ \\
\hline $\begin{array}{l}\text { Numero de } \\
\text { Empalmes } \\
\text { en el enlace }\end{array}$ & $\mathrm{Ne}$ & 8 & und \\
\hline $\begin{array}{l}\text { Margen de } \\
\text { atenuación }\end{array}$ & $\mathrm{Ma}$ & 3 & $\mathrm{~dB}$ \\
\hline $\begin{array}{l}\text { Potencia } \\
\text { del Emisor } \\
(\mathrm{Pi}, \mathrm{Pj}, \mathrm{Pk})\end{array}$ & $\mathrm{Tx}$ & -2 & $\mathrm{~dB}$ \\
\hline
\end{tabular}


Tabla 3. Longitudes de onda asignadas a 16 canales espaciados simétricamente con interferencias fwm sin sobrepasar la penalización $\delta_{\mathrm{FWM}}$.

\begin{tabular}{|c|c|c|c|}
\hline $\begin{array}{l}\text { Canales a } \\
\text { asignarles } \\
\text { frecuencia de } \\
\text { operación }\end{array}$ & $\begin{array}{c}\text { Canal } \\
\text { Utilizado } \\
\text { UIT-T } \\
\text { G.651.1 }\end{array}$ & $\begin{array}{l}\text { Frecuencia } \\
\text { f(THz) }\end{array}$ & $\begin{array}{l}\text { Longitud } \\
\text { de Onda } \\
\lambda(\mathrm{nm})\end{array}$ \\
\hline $\begin{array}{c}\text { CANAL } 1 \\
\text { ASIGNADO = }\end{array}$ & C1 & 195,9375 & 1529,82 \\
\hline $\begin{array}{c}\text { CANAL } 2 \\
\text { ASIGNADO = }\end{array}$ & $\mathrm{C} 2$ & 195,9250 & 1529,92 \\
\hline $\begin{array}{c}\text { CANAL } 3 \\
\text { ASIGNADO = }\end{array}$ & $\mathrm{C} 4$ & 195,9000 & 1530,12 \\
\hline $\begin{array}{c}\text { CANAL } 4 \\
\text { ASIGNADO = }\end{array}$ & C5 & 195,8875 & 1530,22 \\
\hline $\begin{array}{c}\text { CANAL } 5 \\
\text { ASIGNADO = }\end{array}$ & C6 & 195,8750 & 1530,32 \\
\hline $\begin{array}{c}\text { CANAL } 6 \\
\text { ASIGNADO = }\end{array}$ & $\mathrm{C} 7$ & 195,8625 & 1530,42 \\
\hline $\begin{array}{c}\text { CANAL } 7 \\
\text { ASIGNADO = }\end{array}$ & C8 & 195,8500 & 1530,52 \\
\hline $\begin{array}{c}\text { CANAL 8 } \\
\text { ASIGNADO = }\end{array}$ & C9 & 195,8375 & 1530,62 \\
\hline $\begin{array}{c}\text { CANAL } 9 \\
\text { ASIGNADO = }\end{array}$ & C10 & 195,8250 & 1530,72 \\
\hline $\begin{array}{c}\text { CANAL } 10 \\
\text { ASIGNADO = }\end{array}$ & C11 & 195,8125 & 1530,82 \\
\hline $\begin{array}{c}\text { CANAL 11 } \\
\text { ASIGNADO = }\end{array}$ & C12 & 195,8000 & 1530,92 \\
\hline $\begin{array}{c}\text { CANAL 12 } \\
\text { ASIGNADO = }\end{array}$ & C13 & 195,7875 & 1531,02 \\
\hline $\begin{array}{c}\text { CANAL 13 } \\
\text { ASIGNADO = }\end{array}$ & C14 & 195,7750 & 1531,12 \\
\hline $\begin{array}{c}\text { CANAL 14 } \\
\text { ASIGNADO = }\end{array}$ & C15 & 195,7625 & 1531,22 \\
\hline $\begin{array}{c}\text { CANAL 15 } \\
\text { ASIGNADO = }\end{array}$ & C16 & 195,7500 & 1531,32 \\
\hline $\begin{array}{c}\text { CANAL 16 } \\
\text { ASIGNADO= }\end{array}$ & C17 & 195,7375 & 1531,42 \\
\hline
\end{tabular}

Tabla 4. Asignación de 16 canales espaciados simétricamente donde se indica la cantidad de interferencias FWM generadas por canal.

\begin{tabular}{|c|c|c|}
\hline $\begin{array}{c}\text { Para la } \\
\text { asignación } \\
\text { del canal }\end{array}$ & $\begin{array}{c}\text { Canal } \\
\text { utilizado } \\
\text { UIT-T G.651.1 }\end{array}$ & $\begin{array}{c}\mathrm{N}^{\circ} \text { de } \\
\text { tonos o } \\
\text { interferencia } \\
\text { a evaluar }\end{array}$ \\
\hline CANAL 1 & C1 & 0 \\
\hline CANAL 2 & C2 & 0 \\
\hline
\end{tabular}

\begin{tabular}{|c|c|c|}
\hline CANAL 3 & C4 & 0 \\
\hline CANAL 4 & C5 & 12 \\
\hline CANAL 5 & C6 & 24 \\
\hline CANAL 6 & C7 & 56 \\
\hline CANAL 7 & C8 & 108 \\
\hline CANAL 8 & C9 & 184 \\
\hline CANAL 9 & C10 & 288 \\
\hline CANAL 10 & C11 & 424 \\
\hline CANAL 11 & C12 & 596 \\
\hline CANAL 12 & C13 & 808 \\
\hline CANAL 13 & C14 & 1064 \\
\hline CANAL 14 & C15 & 1368 \\
\hline CANAL 15 & C16 & 1724 \\
\hline CANAL 16 & C17 & 2136 \\
\hline
\end{tabular}

Como se puede observar la asignación o búsqueda de un nuevo canal genera interferencias FWM que se incrementan exponencialmente generando un acumulamiento de interferencias en cada canal original asignado.

La acumulación de interferencia FWM producto de la asignación continua de canales genera serias dificultades de evaluación en la penalidad entre el canal 15 y 16 puesto que se evidencia un acercamiento al límite de penalización establecido en $1 \mathrm{~dB}$, por lo tanto la asignación de un canal 17 se dificulta aún más en virtud a que en la evaluación del canal (canal17) se exige evaluar más de 2650 interferencias o tonos FWM representadas en las 2136 que actualmente existen en el sistema, más las 514 interferencias o nuevos tonos que se generarán al adicionar o agregar un nuevo canal con la desventaja que se está evaluando al límite de la penalidad.

El algoritmo evidencia que la generación de estas interferencias es acumulativa y por tanto al agregar un nuevo canal con espaciamiento simétrico, las interferencias existentes se incrementan exponencialmente acercándose rápidamente al límite de penalización. Para evitar que al generar un nuevo canal se alcance el valor de penalización se recomienda asignar un espaciamiento asimétrico entre el canal 16 y el 
canal 17 que permita reducir las interferencias FWM y por lo tanto reducir el acercamiento al límite de penalización FWM que impida la asignación de más canales WDM.

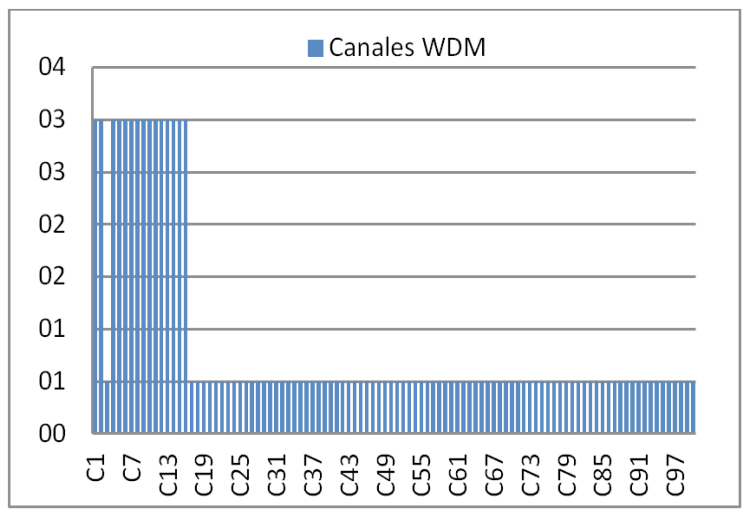

Figura 3. Concentración de canales después de la evaluación del algoritmo propuesto sin espaciamiento asimétrico entre canales.

Teniendo en cuenta la acumulación de la interferencia FWM y la cantidad de tonos generados por este efecto cuando se asignan los canales simétricamente, se establece como parte del algoritmo realizar espaciamientos asimétricos para la asignación de nuevos canales, con lo cual se reducen los tonos de interferencia, la acumulación de la misma y permite tener una mayor disponibilidad de canales para asignar uno nuevo, sin tener un acercamiento rápido al valor de la penalización FWM establecida con la asignación de los primeros 16 canales (figura $3)$.

En la tabla 5 se evidencia el comportamiento y crecimiento de los tonos generados FWM o interferencias durante la asignación asimétrica entre canales.

Como se observa en la figura 4, una vez evaluada la asignación de canales WDM con asignación asimétrica a través del algoritmo planteado se evidencia una mayor concentración de canales habilitados. De igual forma se reducen las acumulaciones de interferencia FWM que permite concentrar los canales de trabajo y posibilitar la asignación de más canales en una grilla 10 veces menor a la establecida inicialmente.
Con los datos y figuras mostradas anteriormente se evidencia que el algoritmo de asignación de longitudes de onda en sistemas WDM funciona de acuerdo a los objetivos propuestos y es aplicable a sistemas de multiplexación WDM

Tabla 5. Asignación de 16 canales espaciados asimétricamente donde se indica la cantidad de interferencias FWM generadas por canal.

\begin{tabular}{|c|c|c|}
\hline $\begin{array}{c}\text { Para la } \\
\text { asignación } \\
\text { del canal }\end{array}$ & $\begin{array}{c}\text { Canal } \\
\text { utilizado } \\
\text { UIT-T G.651.1 }\end{array}$ & $\begin{array}{c}\mathrm{N}^{\circ} \text { de } \\
\text { tonos o } \\
\text { interferencia } \\
\text { a evaluar }\end{array}$ \\
\hline CANAL 1 & $\mathrm{C} 1$ & 0 \\
\hline CANAL 2 & $\mathrm{C} 2$ & 0 \\
\hline CANAL 3 & $\mathrm{C} 4$ & 0 \\
\hline CANAL 4 & $\mathrm{C} 6$ & 4 \\
\hline
\end{tabular}

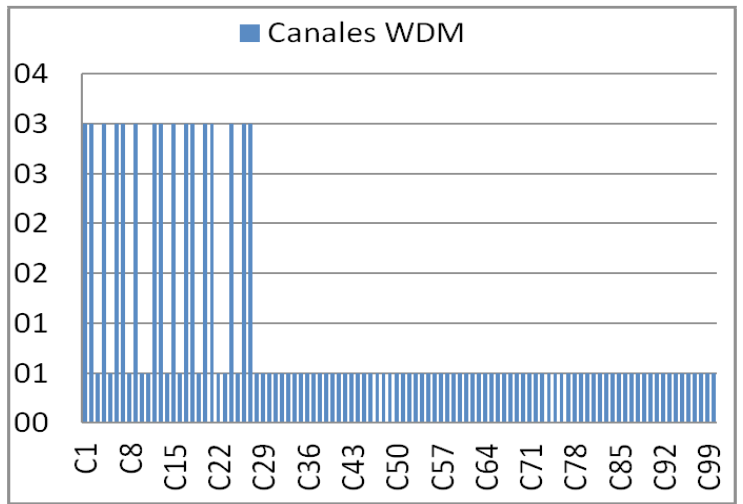

Figura 4. Concentración de canales después de la evaluación del algoritmo propuesto con asignación asimétrica. 


\section{CONCLUSIONES}

Se realizó un compendio de conceptos teóricos y matemáticos que describen todo el comportamiento no lineal de la fibra óptica, su clasificación y dependencia con las características físicas de la fibra y las potencias de las señales trasmitidas.

Se unifican las explicaciones matemáticas y conceptos generados en la literatura para explicar el origen y comportamiento de la no linealidad de generación de la cuarta onda tras haber revisado la literatura que se remonta desde la década de los noventa hasta la actualidad, logrando establecer la formulación matemática base para general nuestro algoritmo.

Se propone un algoritmo ajustado al estándar de la fibra G.652.D para la asignación de longitudes de onda en los sistemas de trasmisión de datos basados en técnicas de multiplexación WDM, partiendo de los modelos y conceptos matemáticos previamente estudiados para describir el mezclado de la cuarta onda, dicho algoritmo permite reducir en promedio un 38\% de las interferencias no lineales generadas

Referencias Bibliográficas

[1] Unión Internacional de Telecomunicaciones. Recomendación ITU-T G.652: Características de un cable de fibra óptica monomodo. 2005.

[2] J. Pereda, A. José; Sistemas y redes ópticas de comunicación, editorial Pearson Education, 2004.

[3] B. Goebel, N. Hanik; Analytical calculation of the number of Four-WaveMixing products in optical multichannel communication system, Technische Universität München,Technical, German,2008. mediante la asignación de canales con espaciamiento asimétrico y aplicables a fibras instaladas en nuestro país. Estándar G.652.D.

El algoritmo propuesto admite asignar canales en sistemas WDM que generan pulsos o tonos FWM sin superar el margen de penalización sugerido para este tipo de interferencias no lineales, incrementando y habilitando en un $40 \%$ el uso de más canales en sistemas WDM especificados por las grillas de la Normas Internacionales (UIT.) Para aplicar en fibras ópticas instaladas en Colombia bajo el estándar UIT-T G.652.D.

El algoritmo planteado establece que para enlaces inferiores a $44 \mathrm{~km}$, la asignación de un nuevo canal está sujeta a un análisis de los productos y tonos FWM que se pueden originar por la inclusión de este nuevo canal, análisis que debe permitir determinar que los nuevos tonos FWM producidos no coincidan o interfieran a canales originales de transmisión ya establecidos, dado que nuestro algoritmo indica que cualquier interferencia generada a longitudes inferiores a $44 \mathrm{~km}$ infringirá las penalizaciones de $1 \mathrm{~dB}$ establecidas para este fenómeno.
[4] K. Inoue, K. Nakanishi, K. Oda; Crosstalk and power penalty due to fiber Four-Wave mixing in multichannel transmissions, IEEE J. Lightwave Technol, Lett, Vol 12, P 1423-1439, 1994

[5] D. Walsh, et al; Practical bit error rate measurements on fiber optic communications links in student teaching laboratories. 9 International Conference on Education and Training in optics and photonics, France, Paper ETOP021, 2005 